\title{
Exploring the chemical reactivity of gallium liquid metal nanoparticles in galvanic replacement
}

\author{
Laia Castilla-Amorós $\dagger$, Dragos Stoian†, James R. Pankhurst $\dagger$, Seyedeh Behnaz \\ Varandili†, Raffaella Buonsanti†*
}

$\uparrow$ Laboratory of Nanochemistry for Energy (LNCE), Department of Chemical Sciences and Engineering, École Polytechnique Fédérale de Lausanne, CH-1950 Sion, Switzerland.

\begin{abstract}
Liquid metals possess intriguing properties and micron/nano-sized particles of these materials are gaining popularity for applications in various research fields. Nevertheless, the knowledge of their chemistry is still very limited compared to other classes of materials. In this work, we explore the reactivity of Ga nanoparticles (NPs) towards a copper molecular precursor to synthesise bimetallic Cu-Ga NPs. Anisotropic Cu-Ga nanodimers, where the two segregated domains of the constituent metals share an interface, form as the reaction product. Through a series of careful experiments, we demonstrate that a galvanic replacement reaction (GRR) between the $\mathrm{Ga}$ seeds and a copper-amine complex takes place. We attribute the final morphology of the bimetallic NPs, which is unusual for a GRR, to the presence of the native oxide shell around the Ga NPs and their liquid nature, via a mechanism that resembles the adhesion of bulk Ga drops to solid conductors. Based on this new knowledge, we also demonstrate that sequential GRRs to include more metal domains are possible. This study illustrates a new approach to the synthesis of Ga-based metal nanoparticles and provides the basis for its extension to many more systems with increased level of complexity.
\end{abstract}




\section{INTRODUCTION}

Liquid metals are an interesting class of materials with fascinating properties deriving from their simultaneous metallic and liquid nature. ${ }^{1-4}$ Besides their use as self-healing contacts in stretchable electronics, liquid metal micron- and nano-sized particles are being explored for biomedical applications, chemical sensors, imaging, and batteries. ${ }^{5-10}$ Shrinking the size of the particles down to the nanoscale adds an extra dimension of complexity which generates new physicochemical properties and opens up new applications. ${ }^{4}$ Ga-based nanoparticles (NPs) are the most studied systems so far. Interestingly, Ga NPs have been demonstrated to possess plasmonic properties that are influenced by their size-dependent solid-liquid transition. ${ }^{11,12}$ Size-dependent plasmonic properties have also been observed for In-Ga NPs in the UV part of the spectrum. ${ }^{13}$ Recently, Ga bimetallic NPs, such as Ga-Pd, Ga-Pt, Ga-Ni, have shown promising catalytic and electrocatalytic performances. ${ }^{9}, 10,14-18$ Theoretical calculations have also predicted that multimetallics including $\mathrm{Ga}$ and $\mathrm{Cu}$, among others, are desirable to improve selectivity in the electrochemical $\mathrm{CO}_{2}$ reduction. ${ }^{16,19,20}$

In order to develop a fundamental understanding of properties at the nanoscale so as to optimise NP performance for targeted applications, synthetic methods that enable size and shape control of NPs are needed. Among these, colloidal chemistry has been demonstrated to achieve superior tunability of metallic NPs with an increasing degree of complexity. ${ }^{21-33}$ However, most of the synthetic development has been carried out on noble metals. ${ }^{21-24,26-33}$ While some of the acquired knowledge within the community can certainly be translated to different systems, new mechanisms might be involved in the formation of non-noble metal NPs. For example, because of their higher oxophilicity, the presence of oxide shells should be taken into account as an important factor during the synthesis. 
In this work, we explore the reactivity of Ga NPs as seeds to synthesise Cu-Ga bimetallic NPs. We find that $\mathrm{Cu}-\mathrm{Ga}$ nanodimers (NDs) form as the reaction products. We combine transmission electron microscopy (TEM), ICP elemental analysis, cyclic voltammetry (CV) and X-Ray absorption spectroscopy (XAS) to investigate the formation mechanism of these anisotropic bimetallic nanostructures. We determine that a galvanic replacement reaction (GRR) takes place and that the peculiar characteristics of the Ga NPs (i.e. their native oxide shell and liquid nature) determine the final ND morphology. Based on this understanding, trimers including Ag-Cu-Ga were also obtained. By demonstrating the applicability of GRR to the construction of multimetallic NPs based on liquid metals, this study paves the way towards future developments of this underexplored class of materials.

\section{EXPERIMENTAL SECTION}

\section{Materials and chemicals}

Tris(dimethylamino)gallium(III) dimer $\left(\mathrm{Ga}_{2}\left(\mathrm{NMe}_{2}\right)_{6}, 99.9 \%\right)$ and di-n-octylamine $\left(\mathrm{C}_{16} \mathrm{H}_{35} \mathrm{~N}\right.$ or DOA, 98\%) were purchased from $\mathrm{ABCR}$. Copper(II) acetate $\left(\mathrm{Cu}(\mathrm{OAc})_{2}, 99.999 \%\right)$, oleic acid $\left(\mathrm{C}_{17} \mathrm{H}_{33} \mathrm{CO}_{2} \mathrm{H}\right.$ or OLAC, $\left.90 \%\right)$, 1-octadecene $\left(\mathrm{C}_{18} \mathrm{H}_{36}\right.$ or ODE, $\left.90 \%\right)$ and oleylamine $\left(\mathrm{C}_{18} \mathrm{H}_{35} \mathrm{NH}_{2}\right.$ or OLAM, $70 \%$ and $\left.80-90 \%\right)$ were all purchased from Sigma-Aldrich. DOA, ODE, OLAC and OLAM were degassed and dried under vacuum at $110^{\circ} \mathrm{C}$ for 4 hours, cooled to room temperature, and then transferred to the glovebox. All syntheses were carried out under an inert atmosphere using anhydrous solvents and standard glovebox and Schlenk-line techniques. Post-synthetic purification ("washing"), handling and storage of the as-synthesised materials was also carried out under inert atmosphere.

\section{Synthesis}

$\mathrm{Cu}-\mathrm{Ga}$ NPs. Ga NPs were synthesised by adapting a literature procedure (details in the Supporting Information). $1 \mathrm{~mL}$ of a solution of these NPs in ODE (4 mM), $1 \mathrm{~mL}$ of a solution 
of $\mathrm{Cu}(\mathrm{OAc})_{2}$ in ODE $(4 \mathrm{mM}), 0.5 \mathrm{~mL}$ of OLAC and $0.5 \mathrm{~mL}$ of OLAM were added to a $5 \mathrm{~mL}$ vial and then stirred at $600 \mathrm{rpm}$ on a hot plate which was set at $150{ }^{\circ} \mathrm{C}$ inside a glovebox for 15 h. Cu-Ga NPs were separated from by-products and from unreacted precursors by adding ethanol ( $3 \mathrm{~mL})$, followed by centrifugation at $5000 \mathrm{rpm}$ for $20 \mathrm{~min}$. The final product was then redispersed in toluene, and the purification/precipitation step was repeated $2-3$ times before finally being stored in anhydrous toluene.

Cu-In NPs. In NPs were synthesised by adapting a literature procedure (details in the Supporting Information). $1 \mathrm{~mL}$ of these NPs in ODE (4 mM), $1 \mathrm{~mL}$ of a solution of $\mathrm{Cu}(\mathrm{OAc})_{2}$ in ODE ( $4 \mathrm{mM}), 0.5 \mathrm{~mL}$ of OLAC and $0.5 \mathrm{~mL}$ of OLAM were added to a $5 \mathrm{~mL}$ vial and then stirred on a hot plate which was set at $150^{\circ} \mathrm{C}$ inside a glovebox for $15 \mathrm{~min}$. The reaction product was separated from by-products and from unreacted precursors by adding ethanol $(3 \mathrm{~mL})$, followed by centrifugation at $5000 \mathrm{rpm}$ for $20 \mathrm{~min}$. Cu-In NPs were redispersed in toluene, and the purification/precipitation step was repeated $2-3$ times before finally being stored in anhydrous toluene.

$A g-G a N P s$. In a typical synthesis, $1 \mathrm{~mL}$ of Ga NP solution in toluene (4 mM) and $1 \mathrm{~mL}$ of a solution of $\mathrm{AgNO}_{3}$ in toluene $(4 \mathrm{mM})$ were added to a $5 \mathrm{~mL}$ vial and then stirred at room temperature for $4 \mathrm{~h}$. The reaction product was separated from by-products and from unreacted precursors by adding ethanol $(2 \mathrm{~mL})$, followed by centrifugation at $5000 \mathrm{rpm}$ for $20 \mathrm{~min}$. AgGa NPs were redispersed in toluene, and the purification/precipitation step was repeated once before finally being stored in toluene.

$A g-C u-G a N P s$. In a typical synthesis, $1 \mathrm{~mL}$ of the $\mathrm{Cu}-\mathrm{Ga}$ nanodimer solution in toluene (2 $\mathrm{mM})$ and $1 \mathrm{~mL}$ of a solution of $\mathrm{AgNO}_{3}$ in toluene $(2 \mathrm{mM})$ were added to a $5 \mathrm{~mL}$ vial and then stirred at room temperature for $8 \mathrm{~h}$. The reaction product was separated from unreacted precursors by adding ethanol $(2 \mathrm{~mL})$, followed by centrifugation at $5000 \mathrm{rpm}$ for $20 \mathrm{~min}$. Ag- 
$\mathrm{Cu}-\mathrm{Ga}$ NPs were redispersed in toluene, and the purification/precipitation step was repeated once before finally being stored in toluene.

\section{Characterisation}

Electron microscopy. Transmission electron microscopy (TEM) images were recorded on an Analytical JEOL-2100F FETEM using a beam energy of $200 \mathrm{kV}$, equipped with a Gatan camera. Samples were drop-cast on a copper TEM grid (Ted Pella, Inc.) prior to imaging. High angle annular dark field-scanning TEM (HAADF-STEM) imaging and energy dispersive Xray spectroscopy (EDXS) were performed on a FEI Tecnai Osiris TEM in scanning mode at an accelerating voltage of $200 \mathrm{kV}$. The microscope is equipped with a high brightness X-FEG gun, silicon drift Super-X EDXS detectors and Bruker Esprit acquisition software.

Inductively Coupled Plasma - Optical Emission Spectroscopy (ICP-OES). Samples were digested with concentrated $\mathrm{HNO}_{3}$ and then diluted for analysis. The concentrations of the nanoparticle solutions were determined on an Agilent 5110 inductively coupled plasma optical emission spectrometry system with a VistaChip II CCD detector.

X-ray Absorption Spectroscopy (XAS). XAS measurements were performed at the SuperXAS beamline at the Swiss Light Source synchrotron facility (Paul Scherrer Institute, Switzerland). A Si(111) channel-cut monochromator was used to condition the beam from the corresponding bending magnet. The beam spot was first focused using a vertically collimating mirror and toroidal focusing mirror to approximately $300 \times 200 \mu \mathrm{m}^{2}$ size. The samples were drop-cast from solution on thin Si wafer substrates inside the glovebox and kept in aluminium bags to avoid exposure to air and light even during the measurements. XAS scans at Ga K-edge $(10.3671 \mathrm{keV})$ and $\mathrm{Cu} \mathrm{K}$-edge $(8.9789 \mathrm{keV})$ were conducted in fluorescence geometry using the PIPS detector (up to 2 spectra per second time-resolution). Both edges were scanned during one event and every scan took around 30 min to get a decent signal for the X-ray absorption 
near edge structure (XANES) spectra. Acquisition of full extended X-ray absorption fine structure (EXAFS) spectra was not possible in this configuration due to the undesired interference from the substrate which led to very intense diffraction peaks. $\mathrm{Cu}$ references $(\mathrm{Cu}$ foil, $\mathrm{Cu}_{2} \mathrm{O}, \mathrm{Cu}(\mathrm{OAc}), \mathrm{CuO}$ - all diluted in boron nitride or cellulose) were acquired in transmission mode. For comparison, we used a $\mathrm{Cu}(\mathrm{OAc})_{2}$ standard from the Farrel Lytle Database. ${ }^{34}$ The reference spectra for $\mathrm{Ga}_{2} \mathrm{O}_{3}$ (in pellet form; diluted in boron nitride) and Gametal (thin-film deposited on weighing paper) were collected in transmission and fluorescence geometry, respectively. Data extraction, normalisation, and averaging (for increased $\mathrm{S} / \mathrm{N}$ ) were performed using the beamline dedicated software for QEXAFS data analysis, ProXAS. ${ }^{35}$ Samples were prepared by drop-casting nanoparticles on low background silicon holders, previously washed with isopropanol and acetone and then preserved under inert conditions by keeping them in sealed aluminium bags (vide supra).

Cyclic voltammetry $(\mathrm{CV})$. The electrochemical measurements were performed in a custommade electrochemical cell using a Biologic SP-300 potentiostat. A glassy-carbon disk was used as the working electrode, a platinum wire as the counter electrode and a Ag wire previously soaked in a concentrated $\mathrm{HCl}$ solution was used as a quasi-reference electrode. The samples were dissolved in an acetonitrile $(\mathrm{MeCN})$ solution of $0.1 \mathrm{M}$ tetrabutylammonium hexafluorophosphate $\left(\left[\mathrm{NBu}_{4}\right]\left[\mathrm{PF}_{6}\right]\right)$ as the electrolyte. Cyclic voltammograms were recorded for quiescent solution containing the samples at variable scan rates and were optimal at $10 \mathrm{mV}$ $\mathrm{s}^{-1}$. All potentials were then referenced against the ferrocenium/ferrocene couple $\left(\mathrm{Fc}^{+} / \mathrm{Fc}=0\right.$ V). In order to compare with the $E^{0}$ of $\mathrm{Ga}$, the potentials were then converted to the Standard Hydrogen Electrode (SHE) scale assuming that $E_{\mathrm{Fc}+/ \mathrm{Fc}}^{0}=0.4 \mathrm{~V}$ vs SHE. ${ }^{36}$ 


\section{RESULTS AND DISCUSSION}

In a typical synthesis of the Cu-Ga NPs (Figure 1a), the Ga seeds are mixed in the reaction vessel with the $\mathrm{Cu}$ precursor (copper(II) acetate, $\left.\mathrm{Cu}(\mathrm{OAc})_{2}\right)$, oleylamine (OLAM) and oleic acid (OLAC) in octadecene (ODE), and then the mixture is stirred for 15 hours under inert atmosphere on a hot plate which was set at $150{ }^{\circ} \mathrm{C}$.

Figure 1 gives an overview of the electron microscopy characterisation of the Ga NPs and of the obtained $\mathrm{Cu}-\mathrm{Ga}$ bimetallic NPs. Figure 1b illustrates the spherical shape and size monodispersity of the starting Ga seeds $(30 \pm 3 \mathrm{~nm}$, Figure S1). The product of their reaction with $\mathrm{Cu}(\mathrm{OAc})_{2}$ is reported in Figure 1c. Mushroom-like nanodimers (NDs), where a spherical domain is interfaced to a drop-shaped domain, are observed. The high angle annular dark fieldscanning TEM (HAADF-STEM) imaging with the corresponding energy dispersive X-Ray spectroscopy (EDXS) mapping (Figure 1d,e) evidences that the spherical domain is $\mathrm{Cu}$ while the drop-shaped domain is Ga. Consistently with this picture, a strong diffraction contrast indicating crystallinity is present only in the $\mathrm{Cu}$ spheres. X-ray Diffraction (XRD) analysis (Figure S2) under an air-free atmosphere confirmed the presence of crystalline metallic copper while no Ga was detected, which is consistent with its amorphous nature in the TEM image. Notably, compared with the initial Ga seeds in Figure 1b, the Ga domain has a decreased size and a deformed shape, and is most narrow near the interface. 


\section{a}
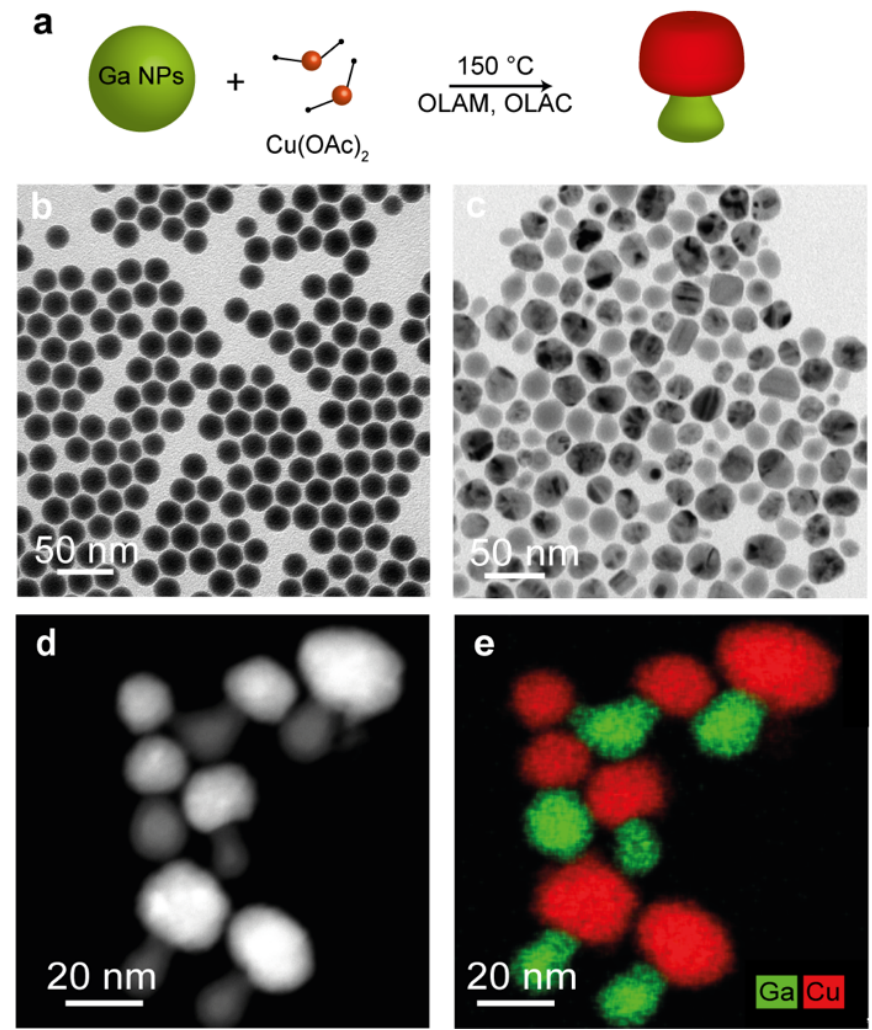

Figure 1. (a) Sketch of the reaction scheme; (b,c) low-resolution TEM image of the as-synthesised Ga NPs and $\mathrm{Cu}-\mathrm{Ga}$ NDs, respectively; (d,e) HAADF-STEM image and corresponding EDXS elemental maps of the NDs, respectively.

To gain further insight into the ND formation mechanism, aliquots were extracted from the reaction mixtures at different times. Figure 2 reports the TEM characterisation of these samples. The images show a progressive reduction of the spherical Ga domain over time, accompanied by growth of the $\mathrm{Cu}$ domain (Figure 2a-e). Longer reaction times result in complete dissolution of the metallic Ga such that only the $\mathrm{Cu}$ domains are clearly detected along with some lower contrast debris (Figure 2f). Consistently with these data, elemental analysis of the supernatant confirmed the increasing concentration of $\mathrm{Ga}$ and decreasing concentration of $\mathrm{Cu}$ as the reaction progressed. A control experiment verified that the reaction conditions alone, in the absence of $\mathrm{Cu}$, do not cause any change in the Ga NPs (Figure S3). 

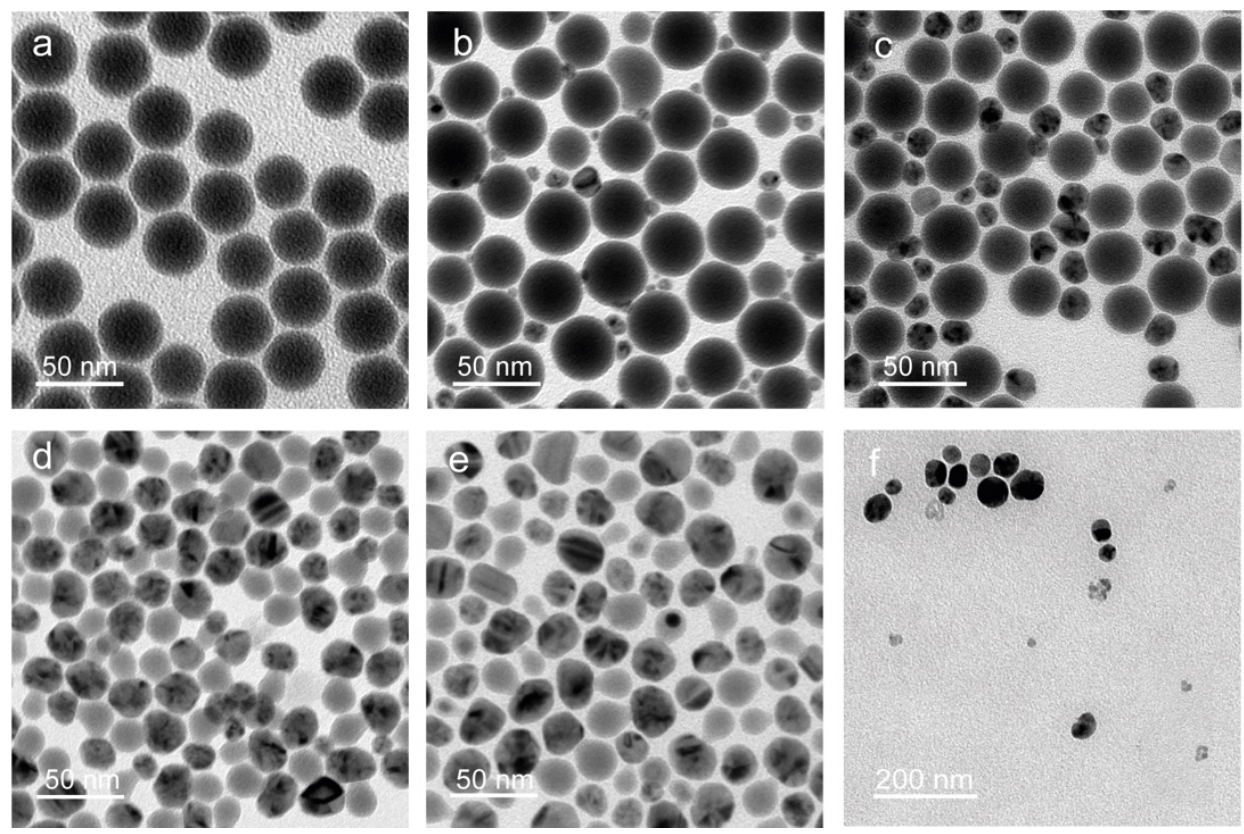

Figure 2. TEM images of samples collected at different times during the synthesis of the Cu-Ga NDs: (a) 0 min, (b) $2 \mathrm{~h}$, (c) $7 \mathrm{~h}$, (d) $11 \mathrm{~h}$, (e) $15 \mathrm{~h}$ and (f) $20 \mathrm{~h}$.

The results above and the standard reduction potentials of $\mathrm{Ga}$ and $\mathrm{Cu}\left(\mathrm{E}^{0}{ }_{\mathrm{Ga}}{ }^{3+} / \mathrm{Ga}=-0.53 \mathrm{~V}\right.$ vs SHE and $E^{0} \mathrm{Cu}^{2+} / \mathrm{Cu}=+0.34 \mathrm{~V}$ vs SHE$)^{37}$ suggest that a GRR might be taking place. GRRs are spontaneous electrochemical processes wherein one metallic domain (the sacrificial template) is oxidised by the cations of another metal that possesses a more positive reduction potential. GRRs have been exploited extensively to synthesise a variety of nanostructured materials; these are mostly based on noble metals but have also been extended to metal oxides more recently. ${ }^{22,38-41}$ Based on these studies and considering that $\mathrm{Cu}$ and $\mathrm{Ga}$ can form solid solutions $^{42}$, NPs with a hollow interior and an alloyed shell are expected as the GRR product.

In order to explain the formation of the atypical ND configuration, first of all we need to assess the contribution of the organic ligands, as examples in the literature show that they can impact the final morphology of the GRR-derived NPs by acting as surface passivating species or coreducing agents. ${ }^{22,43-46}$ Another unique feature of the Ga NPs is their liquid nature and the 
presence of a native self-passivating oxide shell. ${ }^{1-4,11,47,48}$ The fate and role of such an oxide skin during the ND formation also deserves investigation.

Starting with the organic ligands, the simultaneous presence of OLAM and OLAC is needed for the ND to form (Figure S4). OLAM is a well-known reducing agent in the synthesis of NPs in organic solvents ${ }^{49}$, therefore it might contribute to the reduction of $\mathrm{Cu}(\mathrm{OAc})_{2}$ during GRR. It is worth noting that, in the absence of the Ga seeds or in the presence of gallium oxide NPs (Figure S5), no $\mathrm{Cu}$ NPs form under the same reaction conditions, thus OLAM alone is not sufficient for their nucleation. At the same time, if substituted with a stronger reducing agent, such as trioctylphosphine (TOP), $\mathrm{Cu}$ homogeneously self nucleates without any change in the Ga NPs.

One additional consideration is that both OLAM and TOP can coordinate copper ions. ${ }^{25,49}$ While the feasibility of GRRs is normally established based on the standard redox potential of the metals, a different coordination environment can dramatically impact the redox potentials of the $\mathrm{Cu}$ ions in solution. To gain further insight, we studied the redox properties of the complexes that form when $\mathrm{Cu}(\mathrm{OAc})_{2}$ is reacted with only OLAM or TOP (details in the Supporting Information) by performing cyclic voltammetry (CV) experiments (Figure 3). We will refer to these complexes as $\mathrm{Cu}(\mathrm{II})-\mathrm{OLAM}$ and $\mathrm{Cu}(\mathrm{II})-\mathrm{TOP}$. The $\mathrm{CVs}$ of the $\mathrm{Cu}(\mathrm{OAc})_{2}$ and $\mathrm{Cu}$ (II)-OLAM complexes both show two reduction waves; the first is attributed to the $\mathrm{Cu}^{2+} / \mathrm{Cu}^{+}$ reduction and the second, which appears at more negative potential, is attributed to the $\mathrm{Cu}^{+} / \mathrm{Cu}^{0}$ reduction, in agreement with other literature reports. ${ }^{50-52}$ Both peaks are anodically shifted in the OLAM complex compared with $\mathrm{Cu}(\mathrm{OAc})_{2}$. In the $\mathrm{Cu}(\mathrm{II})$-TOP complex, only one peak is observed at $-0.61 \mathrm{~V}$ vs SHE that corresponds to the $\mathrm{Cu}^{2+} / \mathrm{Cu}^{0}$ reduction. Taking the standard redox potential of $\mathrm{Ga}\left(E^{0}{ }_{G a^{3+} / G a}=-0.53 \mathrm{~V}\right.$ vs SHE$)$ as being representative of the Ga NPs, OLAM contributes towards driving the GRR by complexing $\mathrm{Cu}$ and anodically shifting the 
$\mathrm{Cu}^{2+/} \mathrm{Cu}^{+}$redox potential. We speculate that the first reduction causes changes in the $\mathrm{Cu}$-amine complex that enable the complete reduction to metallic $\mathrm{Cu}$. Instead, TOP inhibits the GRR as it renders the $\mathrm{Cu}$ reduction potential more negative than that of $\mathrm{Ga}$.

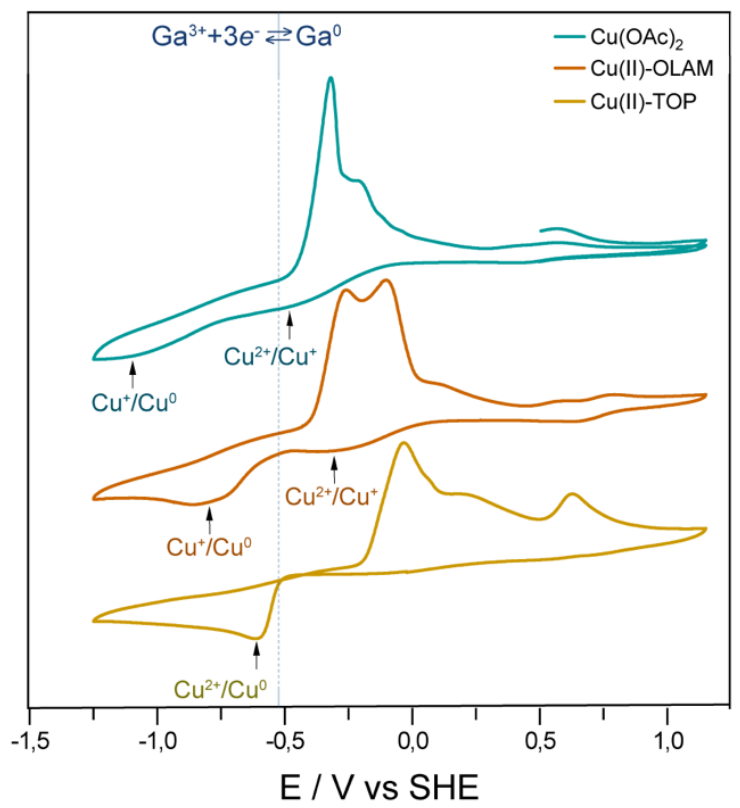

Figure 3. $\mathrm{CVs}$ of $\mathrm{Cu}(\mathrm{OAc})_{2}, \mathrm{Cu}(\mathrm{II})-\mathrm{OLAM}$ and $\mathrm{Cu}(\mathrm{II})-\mathrm{TOP}$, measured in a $\mathrm{MeCN}$ solution of $0.1 \mathrm{M}\left[\mathrm{NBu}_{4}\right]\left[\mathrm{PF}_{6}\right]$ as electrolyte. All potentials were referenced against the ferrocenium/ferrocene $\left(\mathrm{Fc}^{+} / \mathrm{Fc}=0 \mathrm{~V}\right)$ couple and then converted to the SHE scale. The vertical grey-dotted line corresponds to the $E_{G a^{3+} / G a}^{0}$ vs SHE.

As for OLAC, while it is not essential for the reaction to occur, the TEM images suggest that it plays an important role in the control of the morphology (Figure S4). FT-IR and NMR measurements confirm its role as a surface ligand (Figure S6).

Moving towards the investigation of the native oxide shell, Figure 4 provides a summary of its characterisation. EDXS analysis evidences the presence of an oxide shell around both the Ga seeds and the dimers (Figure 4a). Nevertheless, as the samples are exposed to air, albeit only for a few seconds before the holder is inserted into the TEM, the measurement cannot be conclusive alone. Therefore, X-Ray Absorption Spectroscopy (XAS) under inert conditions (details in the Experimental Section) was utilised to elucidate the role of the oxide shell during 
the galvanic replacement. Figure $\mathbf{4 b}$ displays the Ga K-edge XANES spectra on the aliquots taken at different times during the synthesis of the $\mathrm{Cu}-\mathrm{Ga}$ NDs. As the reaction proceeds, a clear shift in the absorption edge position towards higher energy together with an increase in the white-line intensity of the acquired spectra is observed. This trend indicates that the initial Ga NPs are mostly in their metallic form, while by the end of the reaction the signal is dominated by the oxide. Figure $4 \mathbf{c}$ reports the results of a linear combination fitting (LCF) analysis (by using Demeter for XAS data processing ${ }^{53}$ ), that are qualitatively in good agreement with a gradual transition from a metallic to an oxide state. The increasing weight fraction of oxide with time is consistent with the metallic gallium dissolving in solution. As expected, the $\mathrm{Cu} \mathrm{K}$-edge XANES spectra show purely metallic $\mathrm{Cu}$ in all of the aliquots (Figure S7). Altogether the XAS data indicate that the gallium oxide shell is always present throughout the formation of the $\mathrm{Cu}-\mathrm{Ga}$ NDs. A similar conclusion was reached by O'Mullane and collaborators who performed galvanic replacement on bulk Galinstan (GaInSn alloy). ${ }^{15,54}$ 

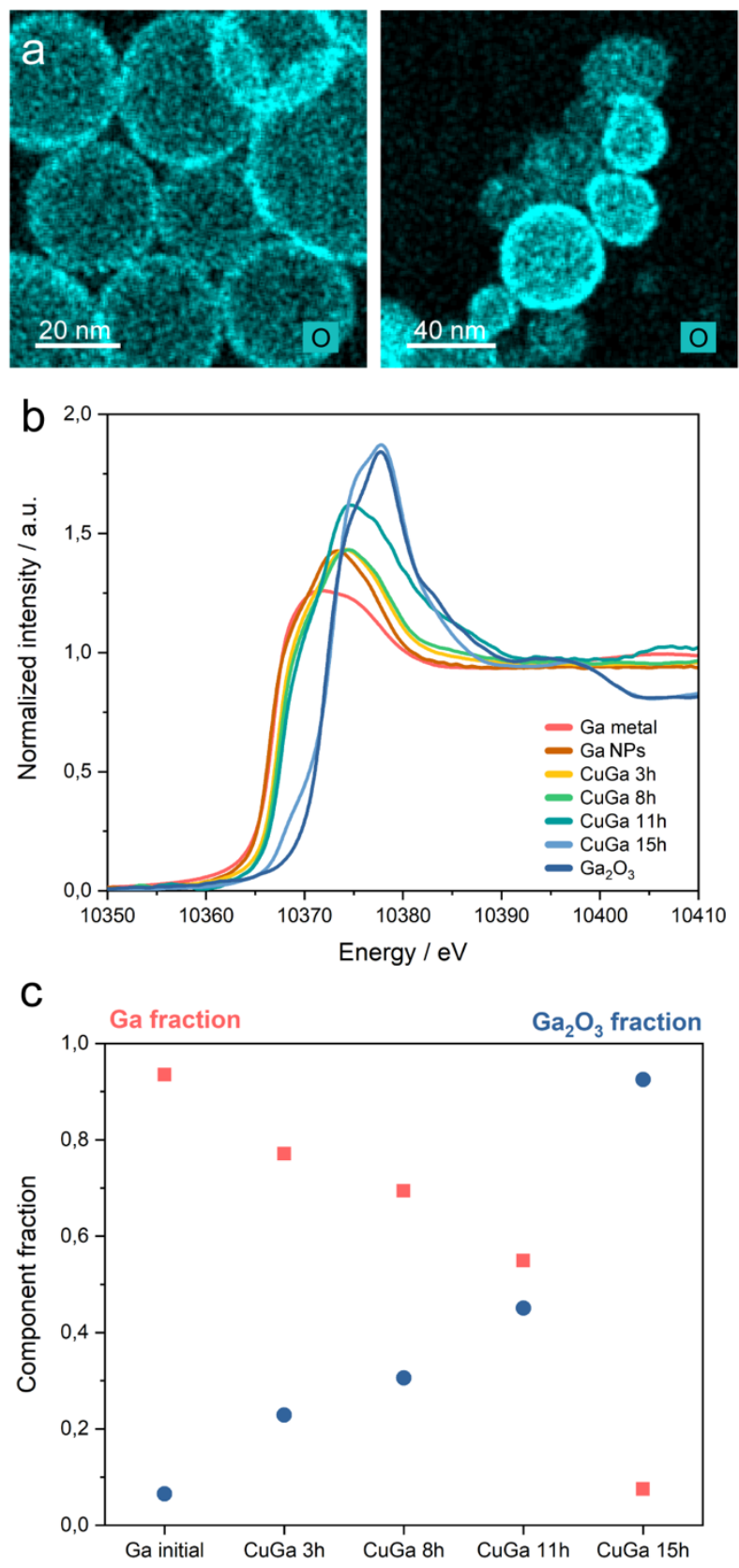

Figure 4. (a) Oxygen EDXS mapping of the initial Ga NP seeds (left) and of the Cu-Ga NDs (right); (b) Ga Kedge XANES spectra on the aliquots extracted at different reaction times. The references for Ga metal (in red) and $\mathrm{Ga}_{2} \mathrm{O}_{3}$ (in blue) are reported; (c) Linear combination fitting (LCF) analysis of the same spectra showing the gradual increasing of the oxidised fraction during the course of the reaction.

Having assessed the role of OLAM as a co-reactant and the presence of the oxide skin throughout the reaction, a mechanistic picture starts to shape up and is sketched in Figure 5. Migration of gallium atoms occurs through the native oxide shell. By analogy with studies on 
liquid metal droplets, such migration can happen because of the shell's mechanical fracturing or because of the establishment of a chemical potential gradient. ${ }^{3,4,15,48,54-56}$ The former is unlikely to be involved in the dimer formation, at least in the initial stages of the reaction, as no external forces are applied, apart from a gentle stirring of the solution. Instead, it is reasonable to assume that the driving force for the gallium migration through the shell is the difference in the reduction potentials of metallic $\mathrm{Ga}$ and the copper-amine complex. The fact that gallium atoms must first migrate through the solid oxide shell justifies the very long reaction times needed for the GRR in $\mathrm{Cu}-\mathrm{Ga}$ compared to those reported for noble metals, which are normally in the range of seconds or minutes. ${ }^{22,26,39,40,46}$ As the Ga reaches the surface, its oxidation coupled with the $\mathrm{Cu}$ reduction takes place. Because of the oxide shell acting as a separator, alloying does not occur. The formation of a core@shell configuration is unfavourable because of the largely different interatomic distances of copper and amorphous gallium oxide. ${ }^{11,57}$ Thus, the $\mathrm{Cu}$ nucleates on the seeds because of the lower activation barrier for heterogeneous nucleation, however continued nucleation of copper on the copper nucleus is then energetically more favourable than nucleation on the gallium oxide surface. This behaviour is similar to that observed in systems where the metal-metal bonding energy between atoms of the newly forming domain is higher than the metal-metal bonding energy between the atoms of the new domain and those of the sacrificial template (i.e. Pt on $\mathrm{Ag}$; $\mathrm{Cu}$ on $\mathrm{Ag}, \mathrm{Au}$, Pt and Pd). ${ }^{58-60}$

As the gallium atoms are consumed in the GRR, one would expect the Ga core to become hollow, as reported for other NPs undergoing GRR. ${ }^{22,38-40}$ Instead a volume reduction is observed and a shape deformation follows as the copper domain sizes increases. In the context of reconfigurable and self-healing electronics, significant efforts are ongoing to study the connection between Ga-based liquid-metal contacts and solid metals, with a prototypical interface being $\mathrm{GaIn} / \mathrm{Cu}^{3,47,48,56,61-65}$ Here, the non-Newtonian rheological properties of the 
gallium oxide skin have been demonstrated to play a significant role as they can mechanically stabilise the liquid core into non-spherical, metastable shapes. ${ }^{3,47,48,56,61-65}$ Furthermore, the oxide interfacial adhesion mechanics when in contact with rigid flat substrates have been shown to cause deformation of the initially spherical liquid metal droplets. ${ }^{3,47,48,56,61-66}$ Similar phenomena might be involved in the formation of the mushroom-like $\mathrm{Cu}-\mathrm{Ga}$ NDs. The increasing deformation of the gallium domain as the copper domain increases in size can be attributed to interfacial adhesion mechanics as well as strain due to the difference in the interatomic distances between the copper and the gallium oxide. The absence of voids in the core and observed reduction in volume of the Ga domain can be explained by the following: the liquid nature of Ga allows the atoms within the oxide skin to redistribute, whilst the oxide skin can adapt to the shrinking volume thanks to its viscoelastic properties.

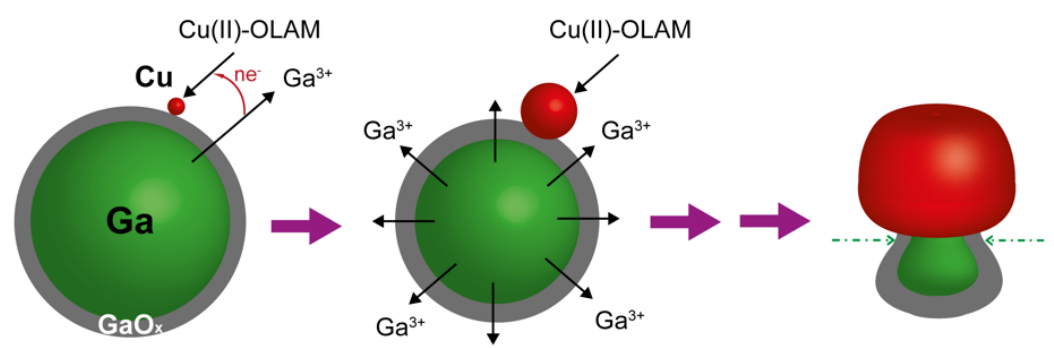

Figure 5. Schematic representation of the proposed formation mechanism of the Cu-Ga NDs.

To prove the importance of the oxide skin and liquid nature of gallium in regulating its chemical reactivity at the nanoscale, we extended the same reaction scheme to other systems (Figure 6). First of all, we substituted $\mathrm{Cu}$ with $\mathrm{Ag}$, a metal with a more positive reduction potential than $\mathrm{Cu}\left(E^{0}{ }_{A g^{+} / A g}=+0.80 \mathrm{~V}\right.$ vs $\mathrm{SHE}^{37}$ and $E^{0}{ }_{C u^{2+} / \mathrm{Cu}}=+0.34 \mathrm{~V}$ vs SHE$)$. Here, the larger potential difference versus $\mathrm{Ga}$ makes the modulation of redox properties via the coordination environment of the cation unnecessary, so OLAM was not needed. Figure 6a evidences that upon reaction with $\mathrm{AgNO}_{3}$, the Ga seeds are decorated by multiple Ag domains. The more similar interatomic distances between Ag and gallium oxide might be one reason for 
the formation of these multimeric NPs instead of dimers. ${ }^{67}$ Importantly, this result confirms that the final morphology of the bimetallic NPs is related to the native oxide and the liquid nature of the Ga seeds and not to the presence of OLAM as a co-reactant.

Secondly, we substituted the Ga NPs with In NPs (Figure S8) as the sacrificial templates during the reaction with $\mathrm{Cu}$. In is another main-group metal with a low melting point $\left(156.6^{\circ} \mathrm{C}\right.$ for bulk), however, it is less oxophilic compared to Ga, meaning that the native oxide shell is not present in the as-synthesised NPs. ${ }^{68-70}$ The standard redox potentials of In and $\mathrm{Cu}$ $\left(E^{0}{ }_{I n^{3+} / I n}=-0.34 \mathrm{~V}\right.$ vs $\mathrm{SHE}^{37}$ and ${E^{0}}_{\mathrm{Cu}^{2+} / \mathrm{Cu}}=+0.34 \mathrm{~V}$ vs SHE$)$ are suitable for galvanic replacement. Figure $\mathbf{6 b}$ shows that bimetallic $\mathrm{Cu}$-In NPs do indeed form. However, here the two metals are mixed, as expected based on the $\mathrm{Cu}$-In phased diagram ${ }^{71}$, rather than segregated as in the $\mathrm{Cu}-\mathrm{Ga}$ NDs. Furthermore, the reaction takes only 15 minutes, which is more reminiscent of a traditional GRR with a metal NP lacking an oxide shell. Overall, these results with In NPs are consistent with the hypothesised role played by the native gallium oxide shell in preventing alloying and in determining the final ND morphology. As a side note, our attempts to remove the amphoteric oxide shell in the Ga NPs or to tune its thickness by acid or base treatments were unsuccessful so far.

Finally, we wanted to explore the possibility of performing sequential GRR reactions on the Ga NPs in order to eventually increase the complexity of Ga-based multimetallic NPs. Figures 6c,d show that Ag-Cu-Ga trimers could be synthesised using $\mathrm{Cu}-\mathrm{Ga}$ NDs as templates. Here future in-depth characterisation and additional experiments will be pursued to understand the delicate interplay between the redox potentials of the three metals, their surface energies and interface energies in determining this final configuration as at the moment it is unclear on which domain nucleation occurs. 

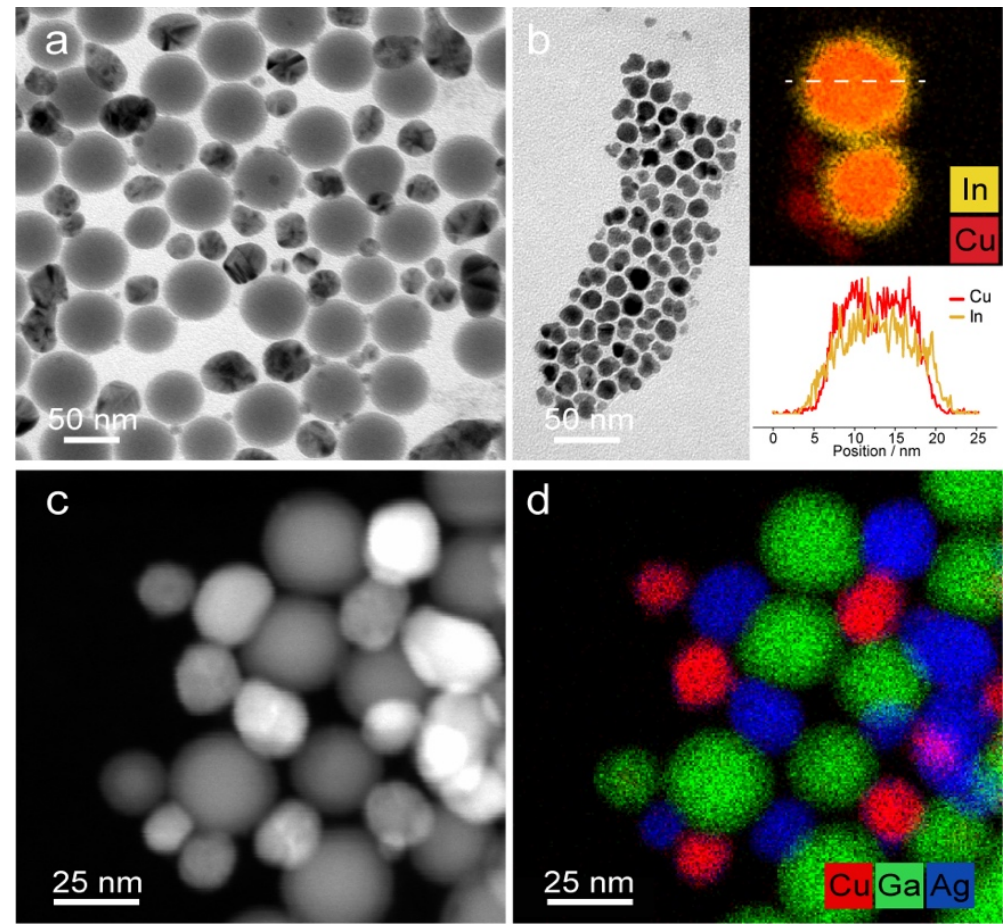

Figure 6. (a) TEM images of the Ag-Ga NPs obtained by reacting Ga NPs with $\mathrm{AgNO}_{3}$; (b) TEM image (left) and EDXS elemental analysis with corresponding line scan (right) of the Cu-In NPs obtained by reacting In NPs with $\mathrm{Cu}(\mathrm{OAc})_{2}$ for only 15 minutes, otherwise the same conditions for the $\mathrm{Cu}-\mathrm{Ga}$ NDs were utilised; (c,d) HAADF STEM image and EDXS elemental map of the Ag-Cu-Ga nanotrimers obtained by sequentially reacting Ga NPs with $\mathrm{Cu}(\mathrm{OAc})_{2}$ and $\mathrm{AgNO}_{3}$.

\section{CONCLUSIONS}

In conclusion, we have explored the reactivity of liquid-metal Ga NPs to synthesise bimetallic Cu-Ga NPs via a colloidal chemistry approach. Investigation of the formation pathway has revealed that a GRR takes place, which is consistent with the redox properties of metallic $\mathrm{Ga}$ and the $\mathrm{Cu}$-amine complex forming in solution. The combination of different characterisation techniques and control experiments has highlighted the key role played by the native oxide shell and liquid nature of the Ga NPs in determining the final nanodimer morphology of the 
bimetallics. Having learned this, the same approach has been applied to access bimetallic AgGa and ternary Ag-Cu-Ga NPs.

Overall, this first study on colloidal liquid metal NPs as sacrificial templates in GRRs showcases the very intriguing and peculiar reactivity of this class of materials at the nanoscale that is certainly underexplored to date. Furthermore, it opens the way towards achieving much more sophisticated and complex structures of Ga-based nanomaterials, which possess promising properties for top-scientific challenges including $\mathrm{CO}_{2}$ electroreduction, sensing, plasmonics, and self-healing electronics. ${ }^{16,19,20}$

\section{ASSOCIATED CONTENT}

The Supporting Information is available and free of charge and contains additional experimental details, information related to XRD experiments, additional TEM, FTIR, NMR and XANES results.

\section{ACKNOWLEDGEMENTS}

This work was primarily financed by the Swiss National Science Foundation (SNSF) under AP Energy Grant, project number PYAPP2_166897/1. DS acknowledges the Sandoz foundation for financial support. JRP and SBV are supported by the H2020 Marie Curie Individual Fellowship grant SURFCAT with agreement number 837378 and by the ERC Starting Grant ERC-HYCAT with agreement number 715634, respectively. The authors thank Dr. Natalia Gasilova for the acquisition of the mass spectrometry data. The authors acknowledge the Paul Scherrer Institut, Villigen, Switzerland for provision of synchrotron radiation beamtime at beamline SuperXAS of the SLS and would like to thank Dr. Adam Clark for assistance. Jan Vavra is acknowledged for discussions on electron microscopy analysis. 


\section{REFERENCES}

(1) Zhang, W.; Ou, J. Z.; Tang, S. Y.; Sivan, V.; Yao, D. D.; Latham, K.; Khoshmanesh, K.; Mitchell, A.; O’Mullane, A. P.; Kalantar-Zadeh, K. Liquid Metal/Metal Oxide Frameworks. Adv. Funct. Mater. 2014, 24, 3799-3807.

(2) Kalantar-Zadeh, K.; Tang, J.; Daeneke, T.; O’Mullane, A. P.; Stewart, L. A.; Liu, J.; Majidi, C.; Ruoff, R. S.; Weiss, P. S.; Dickey, M. D. Emergence of Liquid Metals in Nanotechnology. ACS Nano 2019, 13, 7388-7395.

(3) Daeneke, T.; Khoshmanesh, K.; Mahmood, N.; De Castro, I. A.; Esrafilzadeh, D.; Barrow, S. J.; Dickey, M. D.; Kalantar-Zadeh, K. Liquid Metals: Fundamentals and Applications in Chemistry. Chem. Soc. Rev. 2018, 47, 4073-4111.

(4) Song, H.; Kim, T.; Kang, S.; Jin, H.; Lee, K.; Yoon, H. J. Ga-Based Liquid Metal Micro/Nanoparticles: Recent Advances and Applications. Small 2019, 1903391, 1-21.

(5) Wan, A.; Suchand Sangeeth, C. S.; Wang, L.; Yuan, L.; Jiang, L.; Nijhuis, C. A. Arrays of High Quality SAM-Based Junctions and Their Application in Molecular Diode Based Logic. Nanoscale 2015, 7, 19547-19556.

(6) Lu, Y.; Hu, Q.; Lin, Y.; Pacardo, D. B.; Wang, C.; Sun, W.; Ligler, F. S.; Dickey, M. D.; Gu, Z. Transformable Liquid-Metal Nanomedicine. Nat. Commun. 2015, 6, 1-10.

(7) Chechetka, S. A.; Yu, Y.; Zhen, X.; Pramanik, M.; Pu, K.; Miyako, E. Light-Driven Liquid Metal Nanotransformers for Biomedical Theranostics. Nat. Commun. 2017, 8, $1-19$.

(8) Sun, X.; Sun, M.; Liu, M.; Yuan, B.; Gao, W.; Rao, W.; Liu, J. Shape Tunable Gallium Nanorods Mediated Tumor Enhanced Ablation through Near-Infrared Photothermal Therapy. Nanoscale 2019, 11, 2655-2667.

(9) Esrafilzadeh, D.; Zavabeti, A.; Jalili, R.; Atkin, P.; Choi, J.; Carey, B. J.; Brkljača, R.; O’Mullane, A. P.; Dickey, M. D.; Officer, D. L.; MacFarlane, D. R.; Daeneke, T.; Kalantar-Zadeh, K. Room Temperature CO2 Reduction to Solid Carbon Species on Liquid Metals Featuring Atomically Thin Ceria Interfaces. Nat. Commun. 2019, 10, 18.

(10) Taccardi, N.; Grabau, M.; Debuschewitz, J.; Distaso, M.; Brandl, M.; Hock, R.; Maier, F.; Papp, C.; Erhard, J.; Neiss, C.; Peukert, W.; Görling, A.; Steinrück, H.-P.; Wasserscheid, P. Gallium-Rich Pd-Ga Phases as Supported Liquid Metal Catalysts. Nat. Chem. 2017, 9, 862-867.

(11) Yarema, M.; Wörle, M.; Rossell, M. D.; Erni, R.; Caputo, R.; Protesescu, L.; Kravchyk, 
K. V.; Dirin, D. N.; Lienau, K.; Von Rohr, F.; Schilling, A.; Nachtegaal, M.; Kovalenko, M. V. Monodisperse Colloidal Gallium Nanoparticles: Synthesis, Low Temperature Crystallization, Surface Plasmon Resonance and Li-Ion Storage. J. Am. Chem. Soc. 2014, 136, 12422-12430.

(12) Knight, M. W.; Coenen, T.; Yang, Y.; Brenny, B. J. M.; Losurdo, M.; Brown, A. S.; Everitt, H. O.; Polman, A. Gallium Plasmonics: Deep Subwavelength Spectroscopic Imaging of Single and Interacting Gallium Nanoparticles. ACS Nano 2015, 9, 20492060.

(13) Reineck, P.; Lin, Y.; Gibson, B. C.; Dickey, M. D.; Greentree, A. D.; Maksymov, I. S. UV Plasmonic Properties of Colloidal Liquid-Metal Eutectic Gallium-Indium Alloy Nanoparticles. Sci. Rep. 2019, 9, 1-7.

(14) Armbrüster, M.; Wowsnick, G.; Friedrich, M.; Heggen, M.; Cardoso-Gil, R. Synthesis and Catalytic Properties of Nanoparticulate Intermetallic Ga-Pd Compounds. J. Am. Chem. Soc. 2011, 133, 9112-9118.

(15) Oloye, O.; Tang, C.; Du, A.; Will, G.; O’Mullane, A. P. Galvanic Replacement of Liquid Metal Galinstan with Pt for the Synthesis of Electrocatalytically Active Nanomaterials. Nanoscale 2019, 11, 9705-9715.

(16) Torelli, D. A.; Francis, S. A.; Crompton, J. C.; Javier, A.; Thompson, J. R.; Brunschwig, B. S.; Soriaga, M. P.; Lewis, N. S. Nickel-Gallium-Catalyzed Electrochemical Reduction of CO2 to Highly Reduced Products at Low Overpotentials. ACS Catal. 2016, 6, 2100-2104.

(17) Li, C.; Chen, Y.; Zhang, S.; Zhou, J.; Wang, F.; He, S.; Wei, M.; Evans, D. G.; Duan, X. Nickel-Gallium Intermetallic Nanocrystal Catalysts in the Semihydrogenation of Phenylacetylene. ChemCatChem 2014, 6, 824-831.

(18) Schütte, K.; Doddi, A.; Kroll, C.; Meyer, H.; Wiktor, C.; Gemel, C.; Van Tendeloo, G.; Fischer, R. A.; Janiak, C. Colloidal Nickel/Gallium Nanoalloys Obtained from Organometallic Precursors in Conventional Organic Solvents and in Ionic Liquids: Noble-Metal-Free Alkyne Semihydrogenation Catalysts. Nanoscale 2014, 6, 55325544.

(19) Tran, K.; Ulissi, Z. W. Active Learning across Intermetallics to Guide Discovery of Electrocatalysts for CO2 Reduction and $\mathrm{H} 2$ Evolution. Nat. Catal. 2018, 1, 696-703.

(20) Pedersen, J. K.; Batchelor, T. A. A.; Bagger, A.; Rossmeisl, J. High-Entropy Alloys as Catalysts for the CO 2 and CO Reduction Reactions . ACS Catal. 2020, 10, 2169-2176.

(21) Gao, C.; Goebl, J.; Yin, Y. Seeded Growth Route to Noble Metal Nanostructures. J. 
Mater. Chem. C 2013, 1, 3898-3909.

(22) Xia, X.; Wang, Y.; Ruditskiy, A.; Xia, Y. 25th Anniversary Article: Galvanic Replacement: A Simple and Versatile Route to Hollow Nanostructures with Tunable and Well-Controlled Properties. Adv. Mater. 2013, 25, 6313-6332.

(23) Xia, Y.; Xia, X.; Peng, H. C. Shape-Controlled Synthesis of Colloidal Metal Nanocrystals: Thermodynamic versus Kinetic Products. J. Am. Chem. Soc. 2015, 137, 7947-7966.

(24) O’Brien, M. N.; Jones, M. R.; Brown, K. A.; Mirkin, C. A. Universal Noble Metal Nanoparticle Seeds Realized through Iterative Reductive Growth and Oxidative Dissolution Reactions. J. Am. Chem. Soc. 2014, 136, 7603-7606.

(25) Strach, M.; Mantella, V.; Pankhurst, J. R.; Iyengar, P.; Loiudice, A.; Das, S.; Corminboeuf, C.; Van Beek, W.; Buonsanti, R. Insights into Reaction Intermediates to Predict Synthetic Pathways for Shape-Controlled Metal Nanocrystals. J. Am. Chem. Soc. 2019, 141, 16312-16322.

(26) Anderson, B. D.; Tracy, J. B. Nanoparticle Conversion Chemistry: Kirkendall Effect, Galvanic Exchange, and Anion Exchange. Nanoscale 2014, 6, 12195-12216.

(27) Li, C.; Sato, R.; Kanehara, M.; Zeng, H.; Bando, Y.; Teranishi, T. Controllable Polyol Synthesis of Uniform Palladium Icosahedra: Effect of Twinned Structure on Deformation of Crystalline Lattices. Angew. Chemie - Int. Ed. 2009, 48, 6883-6887.

(28) Li, C.; Shuford, K. L.; Park, Q. H.; Cai, W.; Li, Y.; Lee, E. J.; Cho, S. O. High-Yield Synthesis of Single-Crystalline Gold Nano-Octahedra. Angew. Chemie - Int. Ed. 2007, $46,3264-3268$.

(29) Huang, H.; Wang, Y.; Ruditskiy, A.; Peng, H. C.; Zhao, X.; Zhang, L.; Liu, J.; Ye, Z.; Xia, Y. Polyol Syntheses of Palladium Decahedra and Icosahedra as Pure Samples by Maneuvering the Reaction Kinetics with Additives. ACS Nano 2014, 8, 7041-7050.

(30) Wiley, B.; Herricks, T.; Sun, Y.; Xia, Y. Polyol Synthesis of Silver Nanoparticles: Use of Chloride and Oxygen to Promote the Formation of Single-Crystal, Truncated Cubes and Tetrahedrons. Nano Lett. 2004, 4, 1733-1739.

(31) Sun, Y.; Xia, Y. Shape-Controlled Synthesis of Gold and Silver Nanoparticles. Science 2002, 298, 2176-2179.

(32) Xia, Y.; Xiong, Y.; Lim, B.; Skrabalak, S. E. Shape-Controlled Synthesis of Metal Nanocrystals: Simple Chemistry Meets Complex Physics? Angew. Chemie - Int. Ed. 2009, 48, 60-103.

(33) Zhang, H.; Jin, M.; Xiong, Y.; Lim, B.; Xia, Y. Shape-Controlled Synthesis of Pd 
Nanocrystals and Their Catalytic Applications. Acc. Chem. Res. 2013, 46, 1783-1794.

(34) Boyanov,

B. Farrel

Lytle

Database

http://ixs.iit.edu/database/data/Farrel_Lytle_data/index.html.

(35) Clark, A. H.; Imbao, J.; Frahm, R.; Nachtegaal, M. ProQEXAFS: A Highly Optimized Parallelized Rapid Processing Software for QEXAFS Data. J. Synchrotron Radiat. 2020, 27, 551-557.

(36) Housecroft, C. E.; Sharpe, A. G. Inorganic Chemistry, 4th editio.; Pearson Education Limited, 2012.

(37) Zoski, C. G. Handbook of Electrochemistry, Elsevier.; Amsterdam, 2007.

(38) Sun, Y.; T. Mayers, B.; Xia, Y. Template-Engaged Replacement Reaction: A One-Step Approach to the Large-Scale Synthesis of Metal Nanostructures with Hollow Interiors. Nano Lett. 2002, 2, 481-485.

(39) Yang, Y.; Zhang, Q.; Fu, Z. W.; Qin, D. Transformation of Ag Nanocubes into Ag-Au Hollow Nanostructures with Enriched Ag Contents to Improve SERS Activity and Chemical Stability. ACS Appl. Mater. Interfaces 2014, 6, 3750-3757.

(40) Chen, A. N.; McClain, S. M.; House, S. D.; Yang, J. C.; Skrabalak, S. E. Mechanistic Study of Galvanic Replacement of Chemically Heterogeneous Templates. Chem. Mater. 2019, 31, 1344-1351.

(41) Oh, M. H.; Yu, T.; Yu, S.-H.; Lim, B.; Ko, K.-T.; Willinger, M.-G.; Seo, D.-H.; Kim, B. H.; Cho, M. G.; Park, J.-H.; Kang, K.; Sung, Y.-E.; Pinna, N.; Hyeon, T. Galvanic Replacement Reactions in Metal Oxide Nanocrystals. Science 2013, 340, 964-969.

(42) Liu, S.; Sweatman, K.; McDonald, S.; Nogita, K. Ga-Based Alloys in Microelectronic Interconnects: A Review. Materials 2018, 11, 1-20.

(43) Da Silva, A. G. M.; Rodrigues, T. S.; Haigh, S. J.; Camargo, P. H. C. Galvanic Replacement Reaction: Recent Developments for Engineering Metal Nanostructures towards Catalytic Applications. Chem. Commun. 2017, 53, 7135-7148.

(44) Jing, H.; Wang, H. Structural Evolution of Ag-Pd Bimetallic Nanoparticles through Controlled Galvanic Replacement: Effects of Mild Reducing Agents. Chem. Mater. 2015, 27, 2172-2180.

(45) Polavarapu, L.; Zanaga, D.; Altantzis, T.; Rodal-Cedeira, S.; Pastoriza-Santos, I.; PérezJuste, J.; Bals, S.; Liz-Marzán, L. M. Galvanic Replacement Coupled to Seeded Growth as a Route for Shape-Controlled Synthesis of Plasmonic Nanorattles. J. Am. Chem. Soc. 2016, 138, 11453-11456.

(46) Da Silva, A. G. M.; Rodrigues, T. S.; Slater, T. J. A.; Lewis, E. A.; Alves, R. S.; Fajardo, 
H. V.; Balzer, R.; Da Silva, A. H. M.; De Freitas, I. C.; Oliveira, D. C.; Assaf, J. M.; Probst, L. F. D.; Haigh, S. J.; Camargo, P. H. C. Controlling Size, Morphology, and Surface Composition of $\mathrm{AgAu}$ Nanodendrites in $15 \mathrm{~s}$ for Improved Environmental Catalysis under Low Metal Loadings. ACS Appl. Mater. Interfaces 2015, 7, 2562425632.

(47) Kim, D.; Thissen, P.; Viner, G.; Lee, D. W.; Choi, W.; Chabal, Y. J.; Lee, J. B. Recovery of Nonwetting Characteristics by Surface Modification of Gallium-Based Liquid Metal Droplets Using Hydrochloric Acid Vapor. ACS Appl. Mater. Interfaces 2013, 5, 179185.

(48) Yunusa, M.; Amador, G. J.; Drotlef, D. M.; Sitti, M. Wrinkling Instability and Adhesion of a Highly Bendable Gallium Oxide Nanofilm Encapsulating a Liquid-Gallium Droplet. Nano Lett. 2018, 18, 2498-2504.

(49) Mourdikoudis, S.; Liz-Marzán, L. M. Oleylamine in Nanoparticle Synthesis. Chem. Mater. 2013, 25, 1465-1476.

(50) Rana, M. S.; Rahman, M. A.; Alam, A. M. S. A CV Study of Copper Complexation with Guanine Using Glassy Carbon Electrode in Aqueous Medium. ISRN Electrochem. 2014, $2014,1-7$.

(51) Sandoval-Rojas, A. P.; Ibarra, L.; Cortés, M. T.; Macías, M. A.; Suescun, L.; Hurtado, J. Synthesis and Characterization of Copper(II) Complexes Containing Acetate and N,N-Donor Ligands, and Their Electrochemical Behavior in Dopamine Detection. $J$. Electroanal. Chem. 2017, 805, 60-67.

(52) Elgrishi, N.; Rountree, K. J.; McCarthy, B. D.; Rountree, E. S.; Eisenhart, T. T.; Dempsey, J. L. A Practical Beginner's Guide to Cyclic Voltammetry. J. Chem. Educ. 2018, 95, 197-206.

(53) Ravel, B.; Newville, M. ATHENA, ARTEMIS, HEPHAESTUS: Data Analysis for XRay Absorption Spectroscopy Using IFEFFIT. J. Synchrotron Radiat. 2005, 12, 537541.

(54) Hoshyargar, F.; Crawford, J.; O’Mullane, A. P. Galvanic Replacement of the Liquid Metal Galinstan. J. Am. Chem. Soc. 2017, 139, 1464-1471.

(55) Cabrera, N. .; Mott, N. F. Theory of the Oxidation of Metals. Reports Prog. Phys. 1949, $12,163$.

(56) Tabor, C.; Holcomb, S.; Heikenfeld, J. Reliable and Reversible Contact of Eutectic Gallium Indium and Copper Electrodes. Adv. Mater. Interfaces 2020, 7, 1-8.

(57) Moffitt, S. L.; Zhu, Q.; Ma, Q.; Falduto, A. F.; Buchholz, D. B.; Chang, R. P. H.; Mason, 
T. O.; Medvedeva, J. E.; Marks, T. J.; Bedzyk, M. J. Probing the Unique Role of Gallium in Amorphous Oxide Semiconductors through Structure-Property Relationships. $A d v$. Electron. Mater. 2017, 3, 1-12.

(58) Chen, J.; Wiley, B.; McLellan, J.; Xiong, Y.; Li, Z. Y.; Xia, Y. Optical Properties of PdAg and Pt-Ag Nanoboxes Synthesized via Galvanic Replacement Reactions. Nano Lett. 2005, 5, 2058-2062.

(59) Chen, S.; Jenkins, S. V.; Tao, J.; Zhu, Y.; Chen, J. Anisotropic Seeded Growth of Cu$\mathrm{M}(\mathrm{M}=\mathrm{Au}, \mathrm{Pt}$, or $\mathrm{Pd})$ Bimetallic Nanorods with Tunable Optical and Catalytic Properties. J. Phys. Chem. C 2013, 117, 8924-8932.

(60) Huang, J.; Mensi, M.; Oveisi, E.; Mantella, V.; Buonsanti, R. Structural Sensitivities in Bimetallic Catalysts for Electrochemical $\mathrm{CO} 2$ Reduction Revealed by $\mathrm{Ag}-\mathrm{Cu}$ Nanodimers. J. Am. Chem. Soc. 2019, 141, 2490-2499.

(61) Larsen, R. J.; Dickey, M. D.; Whitesides, G. M.; Weitz, D. A. Viscoelastic Properties of Oxide-Coated Liquid Metals. J. Rheol. (N. Y. N. Y). 2009, 53, 1305-1326.

(62) Ladd, C.; So, J. H.; Muth, J.; Dickey, M. D. 3D Printing of Free Standing Liquid Metal Microstructures. Adv. Mater. 2013, 25, 5081-5085.

(63) Dickey, M. D. Emerging Applications of Liquid Metals Featuring Surface Oxides. ACS Appl. Mater. Interfaces 2014, 6, 18369-18379.

(64) Khondoker, M. A. H.; Sameoto, D. Fabrication Methods and Applications of Microstructured Gallium Based Liquid Metal Alloys. Smart Mater. Struct. 2016, 25.

(65) Xu, Q.; Oudalov, N.; Guo, Q.; Jaeger, H. M.; Brown, E. Effect of Oxidation on the Mechanical Properties of Liquid Gallium and Eutectic Gallium-Indium. Phys. Fluids 2012, 24.

(66) Buonsanti, R.; Grillo, V.; Carlino, E.; Giannini, C.; Curri, M. L.; Innocenti, C.; Sangregorio, C.; Achterhold, K.; Parak, F. G.; Agostiano, A.; Cozzoli, P. D. Seeded Growth of Asymmetric Binary Nanocrystals Made of a Semiconductor TiO2 Rodlike Section and a Magnetic $\gamma$-Fe2O 3 Spherical Domain. J. Am. Chem. Soc. 2006, 128, 16953-16970.

(67) Kumar, S. S.; Rubio, E. J.; Noor-A-Alam, M.; Martinez, G.; Manandhar, S.; Shutthanandan, V.; Thevuthasan, S.; Ramana, C. V. Structure, Morphology, and Optical Properties of Amorphous and Nanocrystalline Gallium Oxide Thin Films. J. Phys. Chem. C 2013, 117, 4194-4200.

(68) He, M.; Protesescu, L.; Caputo, R.; Krumeich, F.; Kovalenko, M. V. A General Synthesis Strategy for Monodisperse Metallic and Metalloid Nanoparticles (In, Ga, Bi, 
$\mathrm{Sb}, \mathrm{Zn}, \mathrm{Cu}, \mathrm{Sn}$, and Their Alloys) via in Situ Formed Metal Long-Chain Amides. Chem. Mater. 2015, 27, 635-647.

(69) Sutter, E.; Sutter, P. Size-Dependent Room Temperature Oxidation of in Nanoparticles. J. Phys. Chem. C 2012, 116, 20574-20578.

(70) Chatterji, D.; West, R. W. Thermodynamic Properties of the System Indium-Oxygen. $J$. Am. Ceram. Soc. 1972, 55, 575-578.

(71) Subramanlan, B. P. R.; Laughlin, D. E. The Cu-In ( Copper-Indium ) System. Bull. Alloy Phase Diagr. 1989, 10, 554-568. 


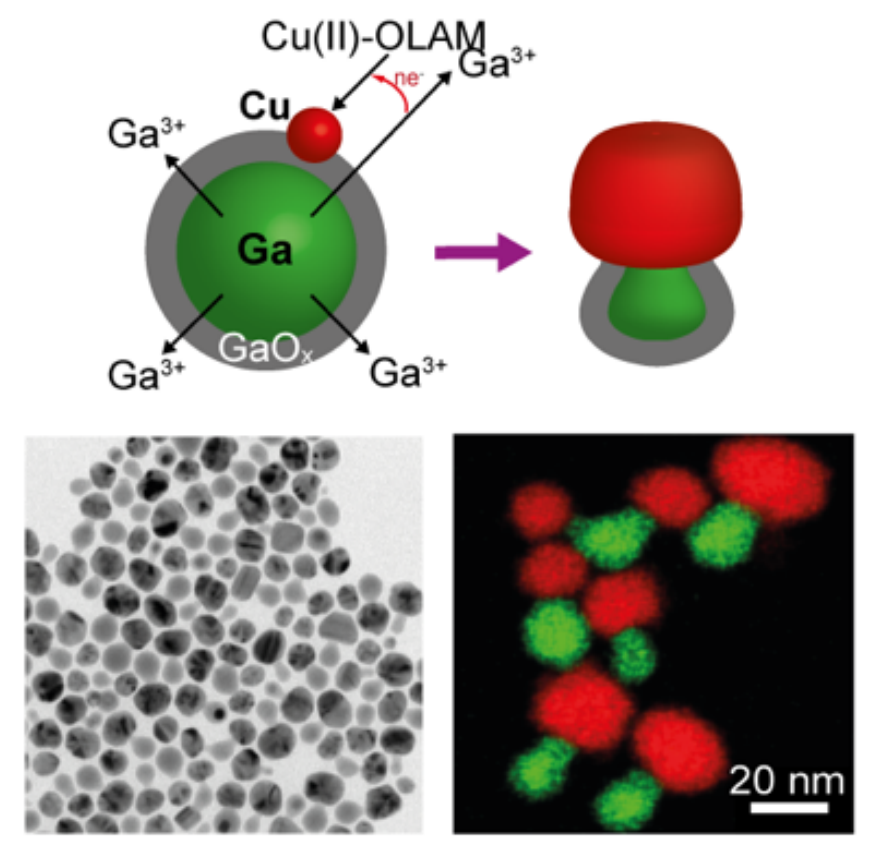

